\title{
Bandgap Engineering of the II-IV, III-V and IV-VI Semiconductor Quantum Dots for Technological Applications
}

\author{
H. I. Ikeri, A. I. Onyia, V. C. Onuabuchi
}

\begin{abstract}
Bandgap engineering in semiconductor quantum dots (QDs) has been studied for technological applications. The model obtained demonstrates that the optical bandgap can be tuned to custom-designed by varying the confinement size. This results in dramatic improvement in band-to-band excitation energy and hence a blue shift in the absorption and luminescence with decreasing QD size. This offers potential revolutionary solutions in many areas of modern science and engineering technology to overcome the fundamental limitation of conventional semiconductors that have their bandgap fixed. In addition, QDs display broad absorption band characteristics with narrow-emission spectra that are tunable due to size quantization effects, which contribute to advancement of medical imaging and multiplexing potentials such as multicolor detection with a single wavelength excitation energy. It is found that CdSe and CdS QDs posses an optical spectrum that confer on them potential active materials for efficient light emitting diode (LED) and lasers operating over the whole range of visible region. ZnS QD possesses the widest bandgap energy which plays a vital role for absorption and emission of high energy blue photons and permits devices to operate at much higher voltages and temperatures crucial for optoelectronic device applications such as Pn junctions and power transistors. In addition, the wide bandgap absorption spectra will be relevant in high optical transmittance specifically in the range of visible to infra red (IR) spectral region. GaAs, InAs and InSb QDs show promising optical bandgap energies in the visible to near infrared (NIR) spectral region which is desirable for optoelectronic devices, operating at NIR wavelengths. We found that $\mathrm{PbS}, \mathrm{PbSe}$ and PbSe QDs displayed exceptional optical characteristics that are favorable for solar cells applications owing to the fact that their absorption band are fairly good match to the solar spectrum.
\end{abstract}

Index Terms - Bandgap Engineering, Quantum Confinement, Quantum Dot, Schrodinger Equation, Spherical Potential Well, Emission and Absorption Spectrum.

\section{INTRODUCTION}

A wide range of semiconductor nano-materials are presently being explored tremendously for their novel applications in optoelectronics, primarily due to the growing need of substituting traditional materials with some alternative cost-effective materials offering commercially viable technologies. For this reason, an increasing amount of effort from both the scientific community and industry has focused on development of novel concepts and theoretical models for controlling the electronic and optoelectreonic properties of semiconductors to meet the specific requirements via bandgap engineering (Smith and Nie, 2010). The process of altering bandgap energies of semiconductors for desired physical properties to meet device applications has driven research into new semiconductor materials leveraging on the principles of quantum confinement to engineer their bandgap and optoelectronics properties to have custum-designed energies, to enhance the performance of photonic devices. This current approach is more relevant, as the conventional.

A common approach to bandgaps engineering is to alloy two or more semiconductors with different bandgaps, and then all bandgaps between the constituent semiconductors can in principle be realized by controlling the alloy composition [1].

In general, the range of bandgaps that can be achieved in high-quality single crystal alloys practically suffers inherent limitations by the strict requirement of lattice matching in planar epitaxial growth, thus the lattice constant of the material to be grown has to match with that of the substrate [2]. Owing to the limited availability of substrates and the lattice mismatch issue, the range of achievable bandgaps is severely constrained and hence not all bandgaps are naturally available through the direct synthesis of elemental or binary individual semiconductors [3]. Revolutionary advancements require semiconductors materials with tunable bandgap characteristics that can be tailored for specific applications. When the material length scales down into the nanometric dimension approximately from $1 \mathrm{~nm}$ to $100 \mathrm{~nm}$ quantum effects become prominent leading to confinement of carriers (electrons and holes) [4], which forces them to occupy space smaller than the normal equilibrium distance in the bulk matter. Precisely, as the size of semiconductors is reduced to nanoscale, their fundamental bandgap energies become size dependent [5] thereby allowing for engineering of their optical and electro-optical responses. Thus decreasing the physical size of a semiconductor into the nanoscale dimension can also be regarded as a bandgap engineering technique as it alters the bandgap due to quantum confinement effects [6]. Such bandgap engineering optimizes their optical performances considerably and provides the state - of - theart device techniques.

An interesting phenomenon which characterizes semiconductors in the nanoscale regime is the ability to control their electrical and optical properties by controlling their sizes known as quantization effect [7], as was demonstrated for quantum structures that have their charge carrier confined in one, two or three directions by potential barriers, such as in quantum wells, quantum wires, and quantum dots respectively, contrary to bulk absorption spectrum that maintains continuous energy band distribution with their charge carriers free to move within their respective bands in all the three directions [8]. The dream materials 
which will enable new applications due to size tunable bandgap has led to the emergence of QDs being the ultimate limit of carrier confinement among other confined structures [9]. The persistence of interest in QDs arises from their novel optical characteristics that are not accessible with the bulk materials [10].

QDs are semiconductors in nanometer dimensions providing strong quantum confinements on the carriers in all the three spatial directions leading to discrete and size dependent energy levels [11]. The confinement of carriers in QDs dramatically modulates the charge carrier states thereby enabling development of new materials with significant milestones in the frontier semiconductor devices [12]. The fundamental bandgap energy of QDs is tunable by varying their confinement dimension which allows engineering of novel materials that have their bandgaps at desired energies [13]. As a result, the bandgaps of these nano-materials can be widely tuned without the detrimental defects that are often unavoidable in bulk materials which are beset with limited tolerance to lattice mismatches. The transport properties of photo excited carriers can be independently and continuously engineered for a given application [14]. This class of nano-materials could have significant potential technological applications on a wide range of photonic and optoelectronic devices, including tunable lasers, solid-state lightings, artificial photosynthesis, light emitting diodes, flat panel displays, Bio-labeling, and new solar cells. In this paper quantum confinement model within the effective mass approximation is extended to analyze the possibility of bandgap engineering in QDs, and their potential applications in different technologies.

\section{BANDGAP ENGINEERING IN QDS VIA QUANTUM CONFINEMENT}

QDs are particles with physical dimensions smaller than the exciton Bohr radius [15]. The small size of QDs leads to "quantum confinement" The quantum confinement effects occur when size of QD is comparable to bulk exciton Bohr radius [16]. The quantum confinement means that the motion of charge carriers is restricted within the quantum box such that the energy levels that the carriers inhabit become discrete, with a finite separation between them [17]. However, there are some regions that the electrons cannot occupy and are collectively known as forbidden region or energy bandgap. The versatility and usefulness of semiconductor materials lies in their fundamental band gap energy which in turn govern their photonics and optoelectronic properties [18]. The bandgap is therefore the most critical parameter for almost all applications involving light absorption and emission [19]. The fundamental bandgap energy of QDs is altered as a function of size due to quantum effect [20]. The optical bandgap is broaden with decreasing particle size leading to a blueshift in the bandgap absorption spectrum, as a consequence, optoelectronic properties can be specifically tuned to have desired energy output by varying the dot size [21].

In a quantum dot, the charge carriers are confined in all three dimensional potential well and by approximation can be described by particle in a sphere in which the photo-excited carriers (electron and hole) may be treated as particles in a spherical potential well [22]. The potential energy is zero everywhere inside the well but is infinite on its walls. We can also refer to this well as a quantum box. The simplest shapes for a three-dimensional box can be that of sphere or a cube. [23]. For a spherical QD of diameter d, the Schrödinger equation is resolved by introducing spherical coordinates and by separating the equation in a radial part and in a part that contains the angular momentum [24]. The potential in this problem is best described mathematically in spherical coordinates, as

$$
V(r, \theta, \emptyset)=\left\{\begin{array}{cc}
0 ; & r \leq L \\
\infty ; & r>L
\end{array}\right.
$$

This describes the region in which the particle can exist as a sphere of radius $\mathrm{L}$ centred at the origin; outside the ball the potential is infinite. Note that this means the potential is independent of $\theta$ and $\phi$. The 3D time-independent Schrödinger equation for this system is

$$
-\frac{\hbar^{2}}{2 \mathrm{~m}} \nabla^{2} \Psi(\mathrm{r}, \theta, \emptyset)=\mathrm{E} \Psi(\mathrm{r}, \theta, \emptyset)
$$

where, $m=$ mass of particle, $E=$ energy of particle, $\Psi=$ wave function associated with particle. Due to the spherical symmetry of the Hamiltonian the wavefunction can be written as $\Psi(\mathrm{r}, \theta, \varphi)=\mathrm{R}(\mathrm{r}) \Theta(\theta) \Phi(\varphi)$, and then the technique of separation of variables results in two differential equations to be solved, one for $\mathrm{R}$ and one for $\mathrm{Y}$. It turns out that the solutions for $\mathrm{R}$ are given by the spherical Bessel functions $\mathrm{Jn}(\mathrm{r})$, and the solutions for $\mathrm{Y}$ are the spherical harmonics $Y_{l m}(\theta, \phi)$. The solution to the spherical well is then

$$
\Psi_{n l m}(r, \theta, \varnothing)=J_{n}(r) Y_{\operatorname{lm}}(\theta, \varnothing)
$$

Since the potential depends on the radius from a fixed point of a spherical quantum dot, the Laplacian $\nabla^{2}$ of the spherical polar coordinate is independent of the angular part and is given as:

$\nabla^{2}=\frac{1}{r^{2}} \frac{\partial}{\partial r}\left(r^{2} \frac{\partial}{\partial r}\right)$

Putting equation (4) into equation (2) for $V=0$ we have

$\frac{-\hbar^{2}}{2 \mu} \frac{1}{r^{2}} \frac{\partial}{\partial r}\left(r^{2} \frac{\partial}{\partial r}\right)=E \Psi(\mathrm{r}, \theta, \varphi)$

The radial part of equation (5) by the method of separation of variables yields:

$$
\frac{1}{R} \frac{d}{d y}\left(r^{2} \frac{d R}{d y}\right)+\frac{2 \mu R y^{2}}{\hbar^{2}}=l(l+1)
$$

where $l=$ orbital angular momentum, the equation (6) is simplified as:

$$
\frac{d^{2}}{d r^{2}}+\frac{2 d R}{r} \frac{d r}{d r}+\left[k^{2}-\frac{n(n+1)}{r^{2}}\right] R(r)=0
$$


where $k=\frac{\mathrm{w} s \mathrm{se}}{\mathrm{z}}$

Equation (7) is reminiscent of spherical Bessel differential equation. The solutions are the spherical Bessel function of order $l_{s} \tilde{f}_{i}(\mathrm{kr})$ and the spherical Neumann function of order $l_{s} n_{l}(k r)$. The general solution is:

$$
R_{n, l}(r)=C_{\mathbb{D}}(k r)+D_{1} n(k r)
$$

where $C_{\mathbb{l}}$ and $D_{\mathbb{l}}$ are constants. Contrary to the spherical Neumann function, the behavior of the Bessel function is such that it is finite at the origin [25]. The finite requirement of the wave function suggests that D must be equal to zero. This simplifies equation (8) to:

$$
R_{n, 1}(r)=C_{D_{1}}(k r)
$$

where, $C_{l}=$ normalization constant and $R_{n \mathbb{l}}=$ eigen function . The wave function must varnish at the boundary. However, due to mathematical expediency, a confining potential that is central was used, which implies that there exist an explicit hard core potential at the centre. But, according to the physical description of the system, no boundary exist at $r=0$, which would introduce a node in the wave function at that point. Rewriting equation (9) in consonance with [26] yields:

$R_{n ! 1}(d)=C_{D_{i}}(k d)$

where, $d=$ diameter of the sphere Also, the infinite potential barrier requires that $\mathrm{R}(\mathrm{d})=0$. This evaluates

$\bar{h}(k d)=0$

wherekd is a zero of the lth-order spherical Bessel function. Unfortunately, the zeros are not located at good points. The boundary condition requires that:

$$
K=\frac{1}{d} X_{n_{d} l}
$$

where $X_{n \mathbb{}}$ is the nth zero of the lth order spherical Bessel function. Comparing equation (12) to $\mathrm{k}$ parameter above yields:

$\frac{1}{d^{2}} X_{n d}^{2}=\frac{2 \mu E}{\hbar^{2}}$

Following equation (13), the allowed energy states $E_{n}$ become

$E_{n}=\frac{\hbar^{2}}{2 \mu d^{2}} X_{n d}^{2}$

Putting $\mu=m_{e}^{*}$ into equation (14) yields the electron confinement energy $E_{e n}$ as:

$E_{e n}=\frac{\hbar^{2}}{2 m_{\alpha}^{2} d^{2}} X_{n_{d} l}^{2}$

wherem $m_{e}^{*}$ is the effective mass of electron. Similarly, the hole confinement energy $E_{k n}$ is obtained as:
$E_{h n}=\frac{\hbar^{2}}{2 m_{h}^{2} d^{2}} X_{n_{d}}^{2}$

where $m_{h}^{*}$ is the effective mass of hole. Adding equations (15) and (16) gives the total confinement energy $E_{c}$ of photo-excited carriers (electron and hole) as:

$E_{c}=\frac{n^{2}}{2 d^{2}}\left(\frac{1}{m_{\varepsilon}^{2}}+\frac{1}{m_{h}^{2}}\right) X_{n_{\mu}}^{2}$

where, $\mathrm{n}=$ radial quantum number that labels the different confined energy states of the particle in the QDs. The ground state corresponds to $n=1$ and $l=0 . X_{1,0}=3.142$ which coincides with the well-known constant $\pi$ [27]. Therefore equation (17) becomes:

$E_{c}=\frac{\pi^{2} h^{2}}{2 d^{2}}\left(\frac{1}{m_{2}^{*}}+\frac{1}{m_{h}^{*}}\right)$

This is confinement energy of a spherical QDs with an electron particle and a corresponding hole in QDs. Electrostatic interaction energy between electron and hole is

$E=\frac{-1.8 e^{2}}{4 \pi \varepsilon_{0} \varepsilon_{r} d}$

where 1.8 is a coefficient due to wave function overlap between the electron and hole in the first excited state whereas the minus signifies the opposite charge polarities that exist between the electron and hole. This term can be fairly significant because the average distance between an electron and a hole in a quantum dot can be small. The last energy term contribution is the bandgap energy of the bulk semiconductor $E_{\text {onmm }}$ which is characteristic of the material. We can now model the size-dependent energy gap of a spherical semiconductor quantum dot as the sum of the bandgap energy of the bulk crystal, confinement energies of electron and the hole and the bound energy of exciton given as

$$
E_{g\left(Q D_{s}\right)}=E_{g_{(b u I l)}}+E_{\text {confinement }}+E_{\text {exciton }}
$$

Then, by inserting equations (18) and (19) into equation 20, we obtain

$$
E_{g\left(Q D_{s}\right)}=E_{g_{(b u I k)}}+\frac{\hbar^{2}}{g d 2}\left(\frac{1}{m_{q}^{*}}+\frac{1}{m_{h}^{*}}\right)-\frac{1.8 \theta^{2}}{4 \pi \varepsilon_{0} \varepsilon_{v} d}
$$

The model predicts that the band gap energy increases as size of the semiconductor QDs decreases. The size-dependent energy gap can be a useful tool for designing materials with well-controlled optical properties. Thus tunable bandgap energies with respect to changes in dot size allows the engineering of bandgap to desired of optical responses for technological applications.

\section{MATERIALS AND METHODS}

The experimental parameters we used for this study are: effective masses of electron, $M_{e}^{*}$ and hole, $M_{h}^{*}$ band gap of bulk

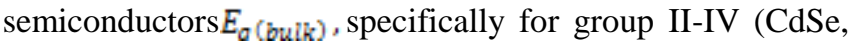


$\mathrm{CdS}$ and $\mathrm{ZnS})$, group III-V (GaAs, InAs and InSb) and the group IV-VI ( $\mathrm{PbSe}, \mathrm{PbS}$ and $\mathrm{PbTe})$ QDs acquired at Advance Physics Laboratory, Sheda Science and Technology Complex Abuja as shown in the Tables 1,2 and 3. The variation of bandgap energies as a function QD size was computed using equation 21 to ascertain the impact of confinement on bandgap engineering. The input parameters used in the theoretical calculation have been listed in Tables 1,2 and 3.

Table 1.The Group II-IV QDs material parameters used for the study.

\begin{tabular}{llll}
\hline Quantum dots & $\boldsymbol{M}_{\varepsilon}^{x}$ & $\boldsymbol{M}_{k}^{x}$ & $\mathbf{E g}_{(\text {bulk }}$ at $300 k$ \\
\hline $\mathrm{CdSe}$ & $0.13 \mathrm{mo}$ & $0.45 \mathrm{mo}$ & $1.74 \mathrm{Ev}$ \\
$\mathrm{ZnS}$ & $0.34 \mathrm{mo}$ & $0.23 \mathrm{mo}$ & $3.68 \mathrm{eV}$ \\
$\mathrm{CdS}$ & $0.21 \mathrm{mo}$ & $0.80 \mathrm{mo}$ & $2.42 \mathrm{eV}$ \\
\hline
\end{tabular}

Table 2.The Group III-V QDs experimental parameters used for the study.

\begin{tabular}{llll}
\hline Quantum dots & $\boldsymbol{M}_{\boldsymbol{\varepsilon}}^{x}$ & $\boldsymbol{M}_{\mathrm{k}}^{x}$ & $\mathbf{E g}_{(\text {bulk) }}$ at 300k \\
\hline InAs & $0.02 \mathrm{~m}_{\mathrm{o}}$ & $0.40 \mathrm{~m}_{\mathrm{o}}$ & $0.36 \mathrm{eV}$ \\
InSb & $0.02 \mathrm{~m}_{\mathrm{o}}$ & $0.40 \mathrm{~m}_{\mathrm{o}}$ & $0.17 \mathrm{eV}$ \\
$\mathrm{GaAs}$ & $0.06 \mathrm{~m}_{\mathrm{o}}$ & $0.51 \mathrm{~m}_{\mathrm{o}}$ & $1.42 \mathrm{eV}$ \\
\hline
\end{tabular}

Table 3.The Group IV-VI QDs experimental parameters used for the study.

\begin{tabular}{llll}
\hline Quantum dots & $\boldsymbol{M}_{\boldsymbol{\varepsilon}}^{x}$ & $\boldsymbol{M}_{\boldsymbol{k}}^{x}$ & $\mathbf{E g}_{(\text {bulk) }}$ at 300k \\
\hline $\mathrm{PbSe}$ & $0.05 \mathrm{~m}_{\mathrm{o}}$ & $0.04 \mathrm{~m}_{\mathrm{o}}$ & $0.27 \mathrm{eV}$ \\
$\mathrm{PbS}$ & $0.25 \mathrm{~m}_{\mathrm{o}}$ & $0.25 \mathrm{~m}_{\mathrm{o}}$ & $0.37 \mathrm{eV}$ \\
$\mathrm{PbTe}$ & $0.17 \mathrm{~m}_{\mathrm{o}}$ & $0.20 \mathrm{~m}_{\mathrm{o}}$ & $0.32 \mathrm{eV}$ \\
\hline
\end{tabular}

\section{RESULTS AND DISCUSSION}

The bandgap energies as a function of dot sizes for group II-IV (CdSe, CdS and $\mathrm{ZnS}$ ), group III-V (GaAs, InAs and $\mathrm{InSb}$ ) and the group IV-VI ( $\mathrm{PbSe}, \mathrm{PbS}$ and $\mathrm{PbTe})$ QDs using the equation 21 are plotted in Figures 3, 4 and 5 respectively. The observed spectra generally indicate substantial increase in bandgap energy and hence the separation between the valence and conduction bands and the band levels become quantized signifying widely spaced energy levels with decreasing crystal size as shown in the Figure 1

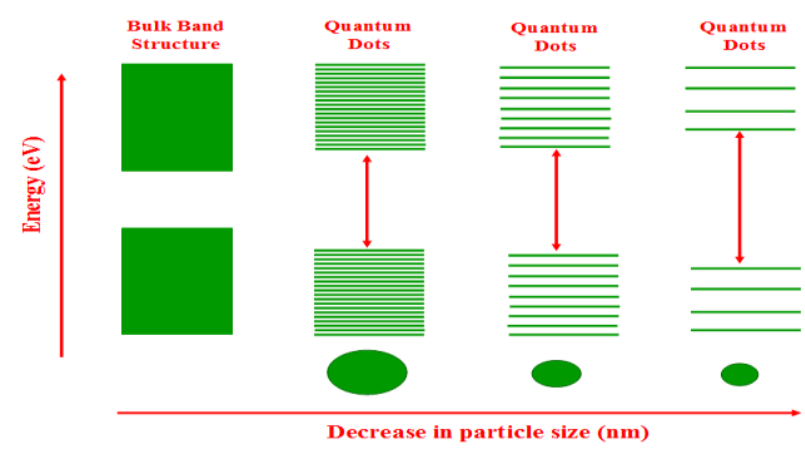

Figure 1: Energy Levels of QDs with Decreasing Size

This results in significant increase in energy of band-to-band excitation peaks and hence a blue shift in the absorption and luminescence bandgap energies as shown in Figure 2 compared with the parental bulks materials that beset with composition-dependent bandgap energy. These optical characteristics suggest that more excitation energy is needed to move electron from valence band to conduction and also more energy will be released during recombination. In this way the electrical properties, optical absorbance and transition energies of the QDs are modulated via size modification.

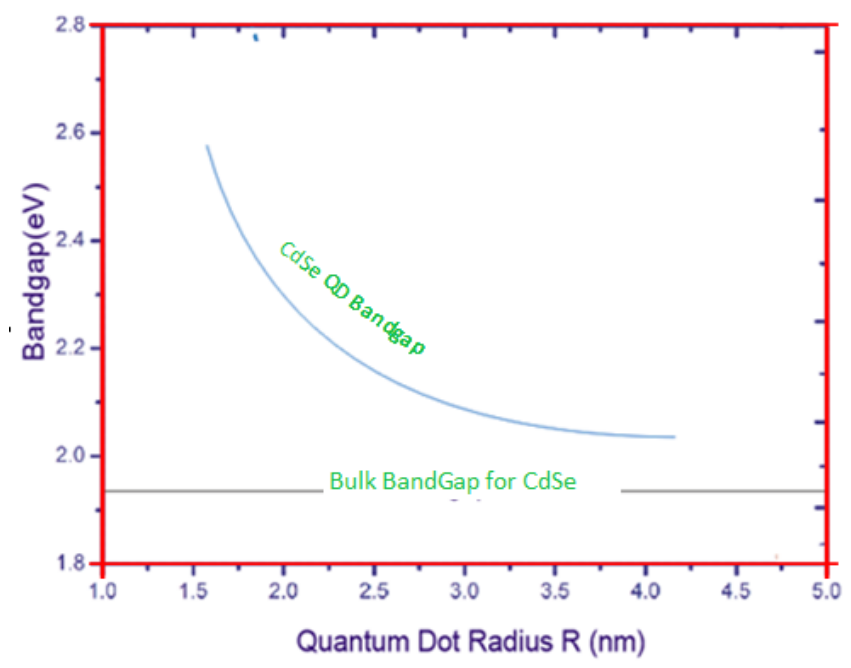

Figure 2: Showing Optical Blue Shift for CdSe QD

The tunable bandgap energies with respect to changes in particle size results in multiple applications. This quantum potential effect on bandgap has significant impact in enhancing performance of photonic devices. A QD that was transparent in infrared region, on increasing band gap may become transparent in visible region. Thus, multi-wavelength photo-detectors would be enabled by semiconductors QDs with such larger range of bandgaps tunability. However, this effect demonstrates the consequences of confining the electrons and electron-hole pair (or the excitons) within a dimension which approaches the critical quantum limit, often termed as the Bohr exciton radius.

It is found that confinement of charge carriers in QDs results in discretization of energy levels and as a result electron transfer can only occur between these discrete electronic states. Consequently, only photon energies of certain dimension can be absorbed leading to monochromatic emissions. Thus QDs can uniquely emit size-dependent single-color fluorescence with high efficiency. In particular, QDs display broad absorption characteristics with narrow-emission spectra that are continuous and tunable due to quantum size effects. The narrow emission and broad absorption bands spectra of the QDs allow single wavelength excitation of emission from different-sized QDs. These fluorescent properties of QDs offer number of advantages that will help to advance biotechnologies in probing several markers at the same time particularly in biosensing/optical imaging and open up several multiplexing potentials such as 
multicolor detection with a single a single wavelength excitation source.

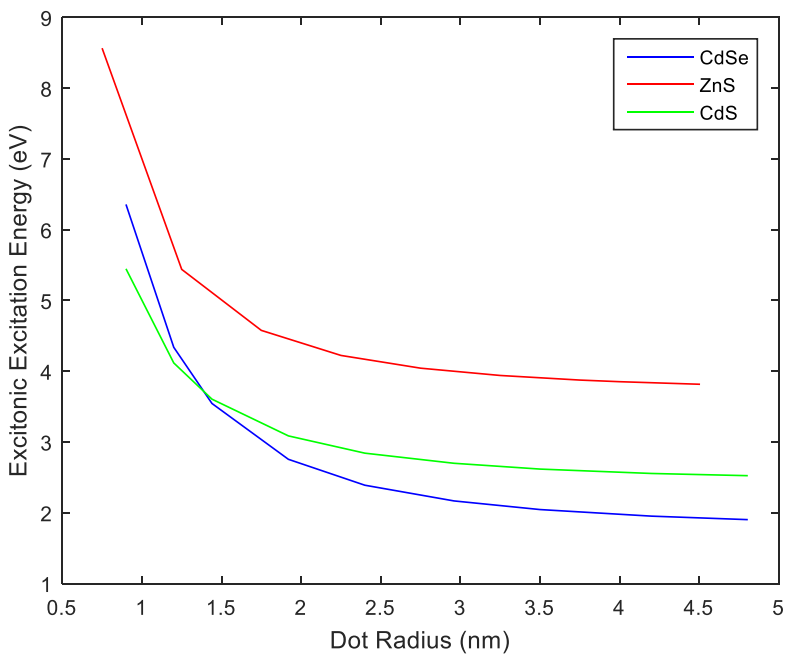

Figure 3: Excitonic Excitation Energy versus Dot Radius for CdSe, $\mathrm{ZnS}$ and CdSQDs

The tunable bandgap energy allows QDs to be optimally engineered for the different applications particularly in photonics and optoelectronics by providing active material for devices. It is apparent from Figure 2 that $\mathrm{CdSe}$ and $\mathrm{CdS}$ QDs posses an optical spectrum that span nearly the entire visible spectrum suitable in solid-state lighting in which illumination or multicolour display is desired. This makes them potential active materials for efficient light emitting diode LED and lasers operating over the whole of visible spectrum. Among group II-IV QD materials considered ZnS possesses the widest bandgap energy and hence the largest tunable spectra range which plays a vital role for absorption and emission of high energy ultraviolet (UV) blue spectrum and permits devices to operate at much higher voltages and temperatures crucial for applications in optoelectronic devices such as Pn junctions and power transistors. In addition, the wide bandgap absorption spectra will be relevant in high optical transmittance specifically in the range of visible to infra red (IR) spectral region.

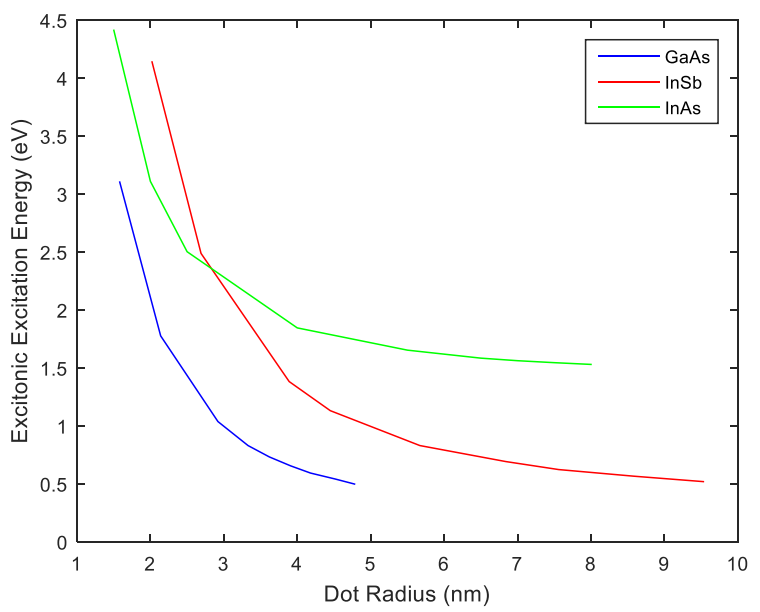

Figure 4: Excitonic Excitation Energy versus Dot Radius for GaAs, InSb and InAsQDs

GaAs, InAs and InSb QDs show promising optical trait in the visible to near infrared (NIR) spectral region which is desirable for optoelectronic applications, including solar cell, dye imaging and light emitting diodes operating at NIR wavelengths.

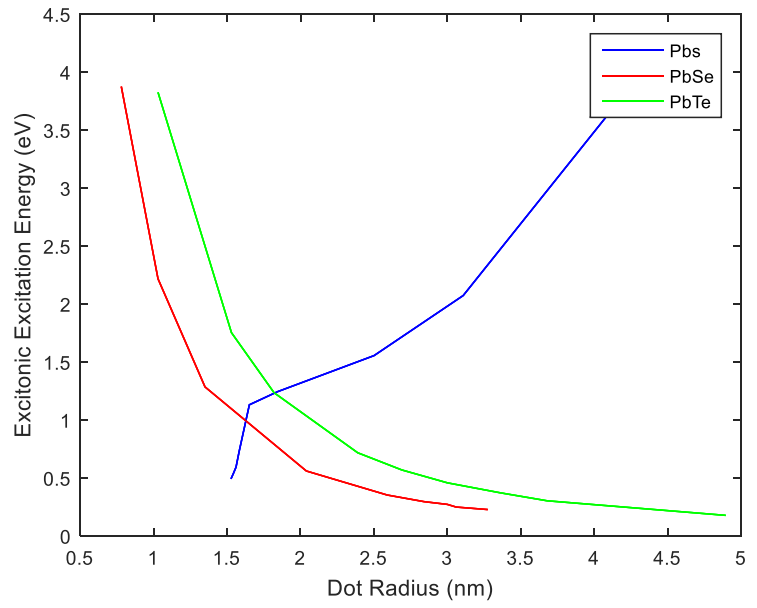

Figure 5: Excitonic Excitation Energy versus Dot Radius for $\mathrm{PbS}, \mathrm{PbSe}$ and $\mathrm{PbSe}$ QDs

We found that $\mathrm{PbS}, \mathrm{PbSe}$ and $\mathrm{PbSeQDs}$ display absolutely exceptional optical characteristics that can be tuned in broad range of UV to IR spectral regions. Solar cell materials selection is largely chosen on the basis of how well their absorption wavelengths match the solar spectrum. This makes $\mathrm{PbS}, \mathrm{PbSe}$ and PbTeQDs suitable materials for solar cell applications owing to the fact that their absorption characteristics are a fairly good match to the solar spectrum.

\section{CONCLUSION}

This work described the successful bandgap engineering in QDs. A simple model is formulated to engineer the band gap energy of semiconductor QDs in different sizes. It is found that the band gap energy of QDs is inversely depends upon the dot size and the band levels become quantized signifying widely spaced energy levels. This produces enhanced optical and electrical properties than that of the bulk materials, and provides opportunities to substantially expand the range of achievable bandgap energies for growing new ranges of materials. One of the most important consequences of the spatial confinement effect observed is an increase in energy of the band-to-band excitation peaks (blue shift) with decreasing dot size in relation with the Bohr radius. Consequently, the electrical properties, optical absorbance and transition energies of the QDs are modulated via size modification The size-dependent absorption and emission spectra with non-zero discrete electronic transition energies in QDs have potential technological applications in optoelectronic devices, including light emitting diodes, solar cells, displays, 
biological labeling and quantum computing etc.

\section{REFERENCES}

[1] V. A. Elyukhin, V. M. Sanchez-R, and O. V. Elyukhina. (2004). Self-assembling in AlxGa1-xNyAs1-y alloys. Applied Physics Letters, 85(10): p. 1704-1706. 3.

[2] L. Zeng, A. Cavus, B. X. Yang, M. C. Tamargo, N. Bambha, A. Gray, and F. Semendy. (1997). Molecular beam epitaxial growth of lattice-matched $\mathrm{ZnxCdyMg1-x-ySe} \mathrm{quaternaries} \mathrm{on}$ InP substrates. Journal of Crystal Growth. 175-176(1): p. 541-545.

[3] H. G. B. Hicks, and D. F. Manley. (1969). High purity GaAs by liquid phase epitaxy. Solid State Communications, 7(20): p. 1463-1465.

[4] Davies, H.(1998). "The physics of low dimensional semiconductors;" an introduction $\left(6^{\text {th }}\right.$ reprint ed.) Cambridge university press.

[5] Augustine Ike Onyia, Henry Ifeanyi Ikeri, Abraham Iheanyichukwu Chima. (2020). Surface and Quantum Effects in Nanosized Semiconductor, American Journal of Nano Research and Applications. Vol. 8, No. 3,pp. 35-41.

[6] Harry S.T., and Adekanmbi M. A. (2020). Confinement Energy of Quantum Dots and the Brus Equation. International Journal of Research -Granthaalayah, 8(11), 318-323.

[7] Madan Singh, Monika Goyal, Kamal Devlal. (2018) Size and shape effects on the band gap of semiconductor compound nanomaterials. Journal of Taibah University for Science,12 (4), $470-475$.

[8] H. Ibach, and H. Lüth. (2003). Solid State Physics. Ed. 3, Springer, Berlin, Germany.

[9] Ikeri, H and Onyia, A. (2019). The dependence of confinement energy on the size of quantum dot. Intl. Jol. of scientific research in Physics and Applied Science, 7(2): 12 - 16.

[10] Li M, Li JC, Jiang Q. (2010).Size-dependent band-gap and dielectric constant of $\mathrm{Si}$ nanocrystals. Int $\mathrm{J}$ Mod Phys B. 24:2297-2301

[11] Ikeri, H. and Onyia, A. (2017). Theoretical investigation of size effect on energy gap of quantum dots using particle in a box model. Chalcogenide Letters, 14(2): 49 - 54.

[12] Jia, G. (2011). Excitons properties and quantum confinement in $\mathrm{CdS} / \mathrm{ZnS}$ core/shell quantum dots.Opt. Adv. Mater, 5, 738-741.

[13] Moreels, I.; Lambert, K.; Smeets, D.; Muynck, D.D.; Nollet, T.; Martins, J.C.; Vanhaecke, F.; Vantomme, A.;Delereu, C.; Allan, G. (2009). Size Dependent Optical Properties of Colloidal PbS Quantum Dots.ACS Nano, 3, 3023-3030.

[14] SapraS, SarmaDD. (2004).Evolution of the electronic structure with size in II-VI semiconductor nanocrystals. Phys Rev B.;69:125304.

[15] Chukwuocha, E., and Onyeaju, M. (2012). Effect of quantum confinement on the wavelength of CdSe quantum dots. International journal of science and technology research, 1 (7), 21-24.

[16] Masumoto Y, Sonobe K. (1997). Size-dependent energy levels of CdTe quantum dots. Phys Rev B.;56:9734-9737.

[17] Segets D, Lucas JM, Taylor RNK. (2012). Determination of the quantum dot band gap dependence on particle size from optical absorbance and transmission electron microscopy measurements. Acs Nano.;6:9021-9032.

[18] Wang, Y.; Tang, Z.; Correa-duarte, M.A.; Liz-marza, L.M.;
Kotov, N.A. (2003). Multicolor Luminescence Patterning by Photoactivation of Semiconductor Nanoparticle Films.J. Am. Chem. Soc, 125, 2830-2831.

[19] Smith AM, NieS. (2010).Semiconductor nanocrystals: structure, properties, and band Gap engineering. AccChemRes.;43:190-200.

[20] Hines MA, Guyot-Sionnest P. (1998). Bright UV-blue luminescent colloidal $\mathrm{ZnSe}$ nanocrystals. J PhysChem B.;102:3655-3657.

[21] Jun YW, Choi CS, CheonJ. (2001).Size and shape controlled $\mathrm{ZnTe}$ nanocrystals with quantum confinement effect. Chem Commun. 2001;(1): 101-102.

[22] Delerue, C. \& Lannoo, M., (2004). Nanostructures: Theory and Modelling. India: Springer.

[23] Viswanatha R, Sapra S, Saha-Dasgupta T. (2005). Electronic structure of and quantum size effect in III-V and II-VI semiconducting nanocrystals using a realistic tight binding approach. Phys Rev B.;72:045333.

[24] Y. Kayanuma. (1988). "Quantum-Size Effects of Interacting Electrons and Holes in Semiconductor Microcrystals with Spherical Shape," Physical Review B, Vol. 38, No. 14, pp. 9797-9805

[25] Weber, H.J., \&Arfken, G.B., (2003). Essential Mathematical methods for Physicist. Academic press, USA.

[26] Dey, S., Swargiary, D., Chakraborty, K., Dasgupta, D., Bordoloi, D., Saikia, R., N..... Choudhury, S. (2012). The Confinement Energy of Quatum Dots. Retrieved on 19th June, 2020 from https://arxiv.org >pdf

[27] Abramowitz, M., \& Stegun, I. (1970). Handbook of mathematical functions with formulas, Graphs and mathematical tables. National bureau of standards.

Ikeri Henry is presently pursuing Ph.D degree in Solid State Physics from Enugu State University of Science and Technology, Enugu, Nigeria. His research work focuses on theoretical characterization and the models for high performance Quantum Dot based on third Generation Solar Cells, under the close supervision of Ass Prof A. I. Onyia. He obtained his M.Sc. Solid State Physics with distinction in 2017 from Enugu State University of Science and Technology, Enugu, Nigeria. His areas of research interest are Nanotechnology, Material Science, Nano Electronics, and Solar cell Technology. He has published a number of research papers in a reputed peer reviewed academic national and international Journals/Conferences. He is working as a Lecturer and currently Head of Department of Electrical/Electronics Engineering, Eastern Polytechnic Port Harcourt, Rivers State, Nigeria.

Ass. Prof. Onyia Ike Augustine did M.Sc. in Physics from Department of Physics and Astronomy, University of Nigeria Nssuka with specialization in Solid State Physics. He completed his Ph.D Solid State Physics in 2016 from Ebonyi State University of Science and Technology Ebonyi, Nigeria. His areas of research interest are Thin Film Fabrication, Nanotechnology, Material Science and Solar Technology. He has published many research papers in reputed, peer-reviewed and citation index local and international journals/conferences. Ass. Prof. Onyia is a senior lecturer of Department of Industrial Physics Enugu State University of Science and Technology ESUT.

V. C. Onuabuchi have B.Sc, and M.Sc degree in Engineering Physics and currently pursuing PhD in Engineering Physics. He is a lecturer in the Department of Industrial physics Enugu State University of Science and Technology. He is an avid researcher who has participated in many national and international conferences/workshops and a member of many scientific organizations. He has published a number of research papers in a reputed peer reviewed academic national and international Journals. His areas of research interest are Material Science, Engineering Physics, Nano Electronics, and Solar cell Technology. 